\title{
Research on Real-time Visual Surveillance Technology of High Speed Mechanical System Movement Features
}

\author{
Jie Zhang $^{1, \text { a }}$, Dong $\mathrm{Xu}^{2}$ \\ ${ }^{1,2}$ Nanchang Institute of Science and Technology, 330108 \\ azhangjiejx@126.com
}

Keywords: high speed machine; movement features; visual surveillance technology

\begin{abstract}
Movement features in high speed mechanical system are showing up quickly aside with the rapid society development. It can not only improve the mechanical movement efficiency, but also determines the whole real-time visual surveillance outcomes. This paper aims at analyzing these features and coming up with optimization solutions.
\end{abstract}

\section{Basic Facts of Real-time Visual Surveillance Technology of High Speed Mechanical System Movement Features}

\section{Real-time surveillance range}

Mechanical engineering technology enters into modern massive production and transportation fields boosting up the production lines and vehicles speed, which can no longer be detected by traditional cameras like turbine engines, airplane propellers, railway wheels and automotive engines movements. Component surface mount adhesives in high speed automatic chip mounters can be completed in few milliseconds. High speed processing and printing are also included. High speed movements in industry can be reflected in a repeat way. As for the movement parts inspection, infrared lasers and speed sensors can be used for components frequency and speed detection. For the complicated application of high speed real time monitoring in human gait and animal behaviors identification, China still needs more work and research on it.

\section{Current situation}

In order to inspect the high speed movement, a high speed visual platform has been developed around domestic and abroad. This platform can record the long-time movement of the experimental rat using the high speed camera and automatically copy the moving routine. It can also be applied into the machine experiment. As for the collecting of animal hearts high speed charts, the adhesive reference points in high speed visual system are available. For the collecting of hearts three-dimensional movement, off-line reconstitution processing and high-speed image processing algorithm are two important tools. By gathering high-speed smart cameras and quickly operating every roll of pictures, smart movement can be found out. Combining with high speed real time detection or extracting high speed videos of feature information and selectivity records, high speed cameras can collect the image sequences then figure out the visual inspection system. The application of high speed cameras can realize several targets one-time collection. The operation of high-speed visual system can be applied to high-speed documents identification and animals real time tracking movements. The faults in liquid crystal displays can be modified in machines.

\section{Visual Inspection System \& Real-time Surveillance System}

\section{High speed visual system}

High speed visual system includes exterior lighting system, personal computer, high-speed image acquisition and processing board and high-speed cameras. During its collecting, the image processing algorithm operating time should be set strictly to male sure the continuation of visual collecting system. FPGA technique would be enough for the simple image processing. The hard algorithm parts in FPGA technique should use the matching methods rather than the hardware language to build up the process speed and form a research direction with high speed visual system. 
In GPU processing, two-way data image transform in GPU and CPU will take up too much time resulting to lost rolls and unable to complete the millisecond algorithm to shape the high-speed curve. So in this research we will need high-speed visual platform, image processing algorithm and image software to realize the real-time calculation.

\section{High speed visual real-time surveillance mechanism}

High speed visual real-time surveillance can clearly record every moment in high speed movement period and fulfill the period characteristics for every pixel points. First we will need the high frame rate pictures and remove the background pixel points through rough filtration to chose the more influential pixel points. Periodic phase values can be reckoned by the original pictures and pixel points. Conducting a true-time monitoring, we can build up the periodic image sample database, vector sample database and phase look-up tables. According to the pixel points in high-speed pictures, matching pictures will be detected in the periodic vector sample database. Match the pictures into the periodic vector phase look-up table to determine the exact roll number and conduct matching calibration work to calculate the true-time high speed periodic movement. The following picture detailed explains the mechanism:

This picture shows a clearer whole inspection structure. Firstly there is a general inspection by the high-speed camera shot. Then combining with the actual situation, periodic sample features processing will be conducted on the off-line images. Finally all kinds of images will be correlated, reset and calculated in intelligent phase form to output the real-time results.

\section{High Speed Visual True-time Monitoring Algorithm}

\section{Rough filtration of original pictures}

High speed periodic images can be gathered in the normal movement. In order to avoid sample data repeat and make sure its adequate, there should have at least five high speed periodic movement in the original pictures recorded the order number of every roll. Rough filtration algorithm can reduce the calculation difficulty in off-line image processing by removing insensitive background pixel points and using the following formula to calculate the time sequence variances of pixel points.

$$
A(x, y)=\sum_{n=1}^{N}\left[I(x, y, n)-\frac{1}{N} \sum_{n=1}^{N} I(x, y, n)\right]^{2}
$$

Comparing with the variances between maximum and minimum numbers, the result will be closer to the real number.

\section{Pixel points detection algorithm in periodic movement features}

High speed visual monitoring needs pixel points in periodic changes to conduct a simple calculation forming a discrete time sequence within the time domain. It also requires every digit in the image sensors storing in the image sequence and forming the pixel point related gray-level periodic curves. According to the obtained time sequence of pixel points, the most varied pixel points in gray value can be found out. Then it ends to the pixel point gathers. According to the specific periodic pixel points, we can draw a definition and select out the distinctive pixel points. More accurate calculations are needed for the general pixel points. Here is the formula:

$$
A_{1}(x, y)=\sum_{n=1}^{N}\left[F(x, y, t(n))-\frac{1}{N} \sum_{n=1}^{N} F(x, y, t(n))\right]^{2}
$$

$\mathrm{A} 1(\mathrm{x}, \mathrm{y})$ stands for the original number in the pixel points inspection. Mean square deviations will be found out after $\mathrm{N}$ times inspections allowing the general pixel points accurately calculated. 
Making sure the basic pixel points, more surveys are needed for the periodic phase value. Normally speaking, the phase value of forward rotation experimental period is $12 \mathrm{r} / \mathrm{s}$. With the system operating, the periodic phase values inspected within 2 seconds shows a monotone decreasing curve. The chart below shows its forward rotation experimental periodic phase image:

\section{Summary}

Real-time visual surveillance technology of high-speed mechanical system movement features acts as a critical role in improving monitoring definition and detailing particular surveillance. In the whole monitoring and inspecting process, the original data detection comes at the first place. With an accurate calculation of periodic changes and operating situation of the phase, we can output the exact phase data to reach an ideal inspection result.

\section{Reference}

[1] Ke Xu, Peng Zhou, Chaolin Yang. Metallic Strip Outside Micro Defects On-line Detection Methods Basing on Photometric Stereo[J]. Journal of Mechanical Engineering. 2013(04).

[2] Qingwu Hu, Zhiyong Chen, Sha Wu. Railway Structural Clearance Rapid Detection Method Basing on Stereo Vision[J]. Journal of the China Railway Society. 2012(01).

[3] Guangqi Hou, Ping Wei, Tingzhu Bai. Research on Multi-channel Embedded High Speed Image Collecting and Storing System[J]. Chinese Journal of Scientific Instrument. 2011(11). 